\title{
Management of constipation in patients with schizophrenia-a case study and review of literature
}

\author{
Ashish Sarangi (DD, Sabiha Armin, Aurelio Vargas, Victoria M. Chu, Kristen Fain and Jessica Nelson
}

\begin{abstract}
Background: Schizophrenia is a disorder that affects about 1\% of the US population, with an extensive impact on patients' health and their risk of later developing comorbidities from treatment. While literature on the side effect profile of antipsychotics is abundant, there are few studies on identification of anticholinergic effects on gut motility and prophylaxis development. The aim of this review is to consider antipsychotic-associated constipation in patients with schizophrenia and to discuss management of antipsychotic-induced constipation as documented in the literature.

Main body: We present a case of antipsychotic-induced constipation and conducted a literature review assessing the prevalence of this issue in this population. The search was done on Embase, MEDLINE, Cochrane Library, and PubMed databases. Key word searches included constipation with concurrent antipsychotic use, antipsychotics and anticholinergic effects, factors causing constipation in schizophrenia, social disparities involved with constipation, and colorectal cancer screenings.

Main findings included high complication rates that may be explained by clozapine-specific side effects, negative health habits, disease, and treatment-related metabolic disorders. Co-existing negative symptoms could also be associated with health outcomes and was found to have adverse consequences on schizophrenia progression. Comorbidities of diabetes and cardiovascular complications contributed to gut hypomotility. Caregiver burden was a factor in delayed recognition of constipation as a side effect. Routine surveillance for symptoms and optimization of medications facilitates early recognition of constipation.

Conclusion: Overall, there is insufficient trial-based evidence to compare the effectiveness and safety of common pharmacological interventions for constipation, such as lactulose, polyethylene glycol, stool softeners, and lubricant laxatives.
\end{abstract}

Keywords: Schizophrenia, Antipsychotic-induced constipation, Constipation screening, Bowel regimen

\section{Background}

Constipation is a common complaint and side-effect for people taking antipsychotics, notably clozapine, thioridazine, chlorpromazine, and olanzapine [1, 2]. Untreated constipation can be severe and lead to complications such as overflow constipation, fecal impaction, paralytic ileus, and death. Among psychiatric patients taking

* Correspondence: aks_sarangi@hotmail.com

Department of Psychiatry, Texas Tech University Health Sciences Center School of Medicine, Lubbock, USA

\section{Springer Open}

antipsychotics, constipation is a symptom that can be overlooked due to the challenges in self-care and health awareness, as well as the patients' higher discomfort or pain threshold [3]. There is insufficient trial-based evidence and literature review on antipsychotic associated constipation in patients with schizophrenia and comorbidities, like hypertension and diabetes, that increase the likelihood of this condition [4]. Appropriate management requires evaluating medical history and medication interactions before recommending a bowel regimen to

(c) The Author(s). 2021 Open Access This article is licensed under a Creative Commons Attribution 4.0 International License, which permits use, sharing, adaptation, distribution and reproduction in any medium or format, as long as you give appropriate credit to the original author(s) and the source, provide a link to the Creative Commons licence, and indicate if changes were made. The images or other third party material in this article are included in the article's Creative Commons licence, unless indicated otherwise in a credit line to the material. If material is not included in the article's Creative Commons licence and your intended use is not permitted by statutory regulation or exceeds the permitted use, you will need to obtain permission directly from the copyright holder. To view a copy of this licence, visit http://creativecommons.org/licenses/by/4.0/. 
this population. For patients taking calcium channel blockers in addition to antipsychotics, there may be a synergistic effect on slowing of bowel motility that necessitates prophylactic bowel regimen to reduce medication noncompliance and hospitalizations $[5,6]$.

Dysregulation of dopaminergic neuronal function is the central pathophysiology to psychosis. Dopamine hyperactivity in the mesolimbic and hypoactivity in the mesocortical pathway are hypothesized to be the manifestation of positive and negative symptoms seen in schizophrenia and can be managed with first (FGAs) or second (SGAs) generation antipsychotics [1-3]. Highpotency FGAs exhibit greater extrapyramidal side effects due to greater antagonism to the D2 receptor while lowpotency FGAs exhibit greater anticholinergic effects and antihistaminergic. SGAs are comparable to FGAs in clinical efficacy without increasing the risk for extrapyramidal side effects, due to their role in 5HT2A receptor modulation [6, 7]. It is recognized, however, that SGAs differ in affinity, dissociation rates, and specificity of brain areas for D2 receptor affinity when compared to FGAs, leading to significantly less D2 blockage side effects and complications over time [6, 7].

Central side effects can be difficult to recognize in psychiatric patients as symptoms often overlap with clinical disorders (i.e., confusion and delirium). Regardless of the duration, central anticholinergic side effects can further impair memory, concentration, and confusion in comorbid psychiatric conditions especially among people with schizophrenia [8]. Furthermore, the concurrent use of antipsychotics and other drugs with anticholinergic profiles (i.e., antihistamines, antidepressants, calcium channel blockers, antispasmodics) may further exacerbate anticholinergic side effects leading to greater impairment of cognitive functioning. Anticholinergic side effects are prone to being underreported by psychiatric patients due to communication difficulties, sedation and analgesic properties of medical regimens, or limited awareness of acute/chronic symptom presentation [9]. Gastrointestinal hypomotility is a well-documented effect of anticholinergic drugs and if unrecognized, gastrointestinal hypomotility may progress into a medical complication as in the case of constipation or impact ileus [1-4]. While some FGAs exhibit anticholinergic properties, i.e., haloperidol, thioridazine, and chlorpromazine; SGAs show greater affinities for cholinergic receptors in vitro [10]. Among the atypical antipsychotics, clozapine, quetiapine, and olanzapine demonstrate significant muscarinic receptor affinity in vitro that correlates in a dose dependent manner [9-11]. Systematic reviews demonstrate patients taking clozapine have significantly higher rates of antipsychotic induced constipation vs patients on other antipsychotics, showing as many as $60 \%$ of patients developing constipation [4].

\section{Main text}

\section{Case study}

A 46-year-old male with schizoaffective disorder, hypertension, and type II diabetes mellitus presented to the emergency center with acute psychosis and aggressive behavior after stopping his medications (haloperidol, valproate, diphenhydramine) for 2 months. In the interview, the patient did not exhibit psychosis symptoms or aggression; his chief complaint was the stomach pain, nausea, diarrhea, and constipation that may have been precipitated by eating bad food. He reports medication noncompliance due to side effects of nausea and dry mouth. Lab-work on admission showed $\mathrm{CBC}$, CMP, and TSH/T4 within the normal limits; urine drug screen toxicology and urinalysis were negative. On a physical exam, the patient's abdomen was distended and rigid. A kidney-ureter-bladder and abdominal X-ray revealed increased stool burden as the probable source of the patient's abdominal pain. The patient was subsequently treated with magnesium hydroxide and polyethylene glycol. We restarted the appropriate treatment for the patient's schizophrenia and hypertension, with improvement of the patient's condition. A prophylactic bowel regimen of docusate sodium was recommended upon discharge, in addition to his other medications (haloperidol, valproate, diphenhydramine, and amlodipine). During the history taking, the patient denied any concerning symptoms for constipation despite abdominal distention. Given the risk factors and physical findings, it was useful to follow an algorithm such as Fig. 1, to obtain proper imaging and begin a trial of laxatives.

This case highlights the need to maintain a high index of suspicion for anticholinergic side-effects secondary to medication interaction, especially in a patient group that exhibits diet and lack of exercise related risk factors to slowed motility. Rapid diagnosis and early treatment are crucial for the health outcomes for people with schizophrenia and other comorbidities. The outcome of this case showed good resolution of constipation when treated with laxatives. Since the patient did not have a history of non-compliance due to constipation, we advised him to keep track of his diet and symptoms as well as encouraged the use of laxatives if similar episodes occur.

\section{Challenges in recognizing constipation as a treatment- limiting side-effect}

Successful schizophrenia treatment depends on a combination of factors as current medications help with paranoia, delusions, and hallucinations, but may not address social withdrawal and other negative symptoms. Tools to manage a challenging disorder such as schizophrenia, such as building a strong support system and implementing health strategies with diet modification, 


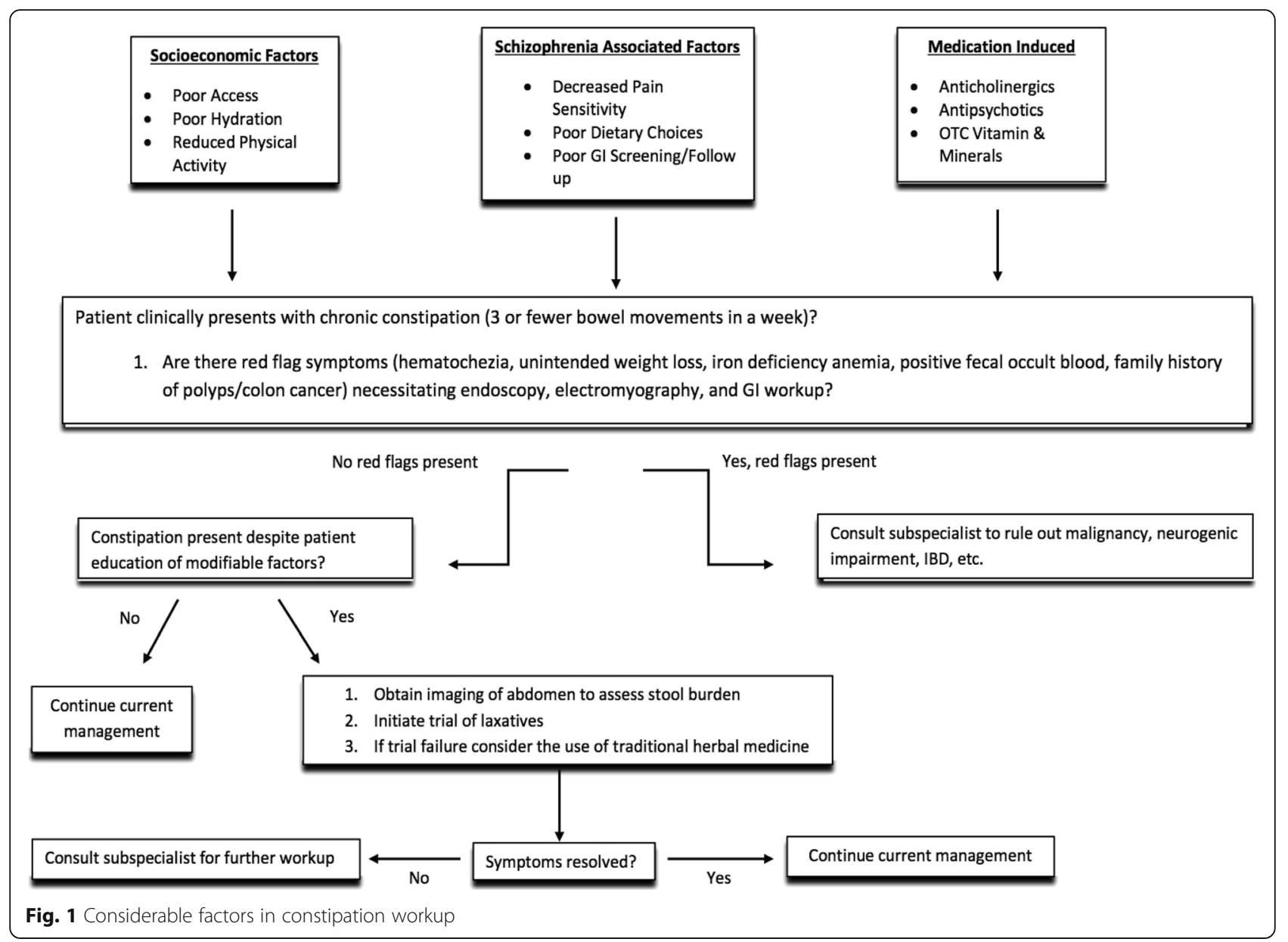

stress relief, and exercise regime can have a profound effect on the frequency and severity of symptoms and reduce the likelihood of developing comorbidities that add to the disease burden $[7,12,13]$.

Patients with this disorder have been observed to have poor nutritional statuses which can contribute to constipation issues, such as limited intake of healthy lipids, fiber, proteins, and vitamins [13, 14]. Compared to healthy cohorts' levels, schizophrenic patients are at risk of lower folate and Vit B12 levels, with only $10.7 \%$ of 428 schizophrenic patients reporting healthy dietary patterns [14]. Another study in nutritional neuroscience found that more than half the studied schizophrenic patients had food addiction, which in term increased the risk of obesity and cardiovascular risk [15]. Kim et al, found significantly lower dietary intakes of protein, polyunsaturated fatty acids (PUFAs), vitamin $\mathrm{K}$, niacin, folate, and vitamin $\mathrm{C}$ in male schizophrenic patients compared to the male control subjects [16]. Additionally, protein deficiency has been linked to reduced cognitive functioning, independent of demographic or environmental factors [17].
While the impact of various antipsychotics on metabolic syndrome and their possible mechanisms are comprehensively reviewed, most are case reports that specifically discuss clozapine-induced gut hypomotility and life-threatening ileus [18-22]. Patients using antipsychotics had a 1.9 higher risk of developing constipation compared to non-users. Stanniland and Taylor reported rates of 1.5 to $25 \%$ compared to placebo [23]. With clozapine use versus that of other medications, constipation is one of the top 4 reported side effects with rates ranging from 14 to $60 \%$ [18-22]. Other antipsychotics have varying anticholinergic effects, as seen in Table 1.

Palmer et al. reports that clozapine-induced gastric hypomotility is often silent, with self-reported constipation having low sensitivity in its diagnosis, even using the Rome criteria, and treating gastric hypomotility based on self-reported symptoms questions will miss most cases [21]. Virtanen et al. found that constipation and dyspepsia are gastrointestinal symptoms that are often ignored in research on physical comorbidities of schizophrenia, with the prevalence of constipation at $31.3 \%$ and of dyspepsia 23.6\% [24]. Some risk factors for 
Table 1 Antipsychotic formulations and side-effect profiles

\begin{tabular}{llll}
\hline Name & Route & Likelihood of Induced Constipation & Notes \\
\hline Olanzapine & IM & ++ & Constipation secondary to weight gain, metabolic issues \\
Clozapine & PO & & \\
Chlorpromazine & PO & +++ & Constipation secondary Metabolic issues \\
Thioridazine & PO & ++ & Anticholinergic, Atony of colon \\
& PO & +++ & Anticholinergic \\
\hline
\end{tabular}

++ moderate/occurs frequently, +++ severe/occurs most of the time

constipation were paracetamol and clozapine use, older age, and living in sheltered homes. Most reports are about the severe and often fatal gastric ileus induced by clozapine and include some discussion on bowel regimens for management, but our review shows that there is little consensus on early diagnosis criteria and recommendations on bowel regimen even though the anticholinergic side effect profile of antipsychotics is known.

Furthermore, people with schizophrenia can have a difficult time addressing constipation due to issues with self-care, often neglecting to practice healthy behaviors such as exercise and weight control, resulting in greater occurrence of metabolic and anticholinergic side-effects because of antipsychotic use [25, 26]. Disturbances in thoughts, behavioral changes, and impaired cognitive functions which affect a person's ability to work or communicate socially and incapability of self-care characterize schizophrenia $[27,28]$. These features make it difficult for the affected individual to carry out daily activities and maintain personal hygiene. Poor health status in those with schizophrenia has been attributed to factors like poverty, less resources, medications, and more so, the effect of systemic diseases like diabetes, hypertension, and osteoporosis which reduce the selfcare ability of the patients [26-28]. In a study conducted by Tas et al., it was found that patients admitted to an in-patient facility for "failure to meet basic needs" need assistance in the early discharge period for parameters such as "coping, need of knowledge, treatment and care, self-care, sleep, role and relationships." [27] ÇiftÇi et al. found that the average self-care ability among schizophrenia patients was lower than that of the individuals with anxiety disorder and depression [28]. The lack of some qualities that maintain self-care, such that lack of physical energy, awareness, decision-making, motivation, and confidence, can decrease the self-care ability of schizophrenic patients.

Additionally, mental health experts have recently discussed a challenge to proper gastrointestinal pathology screening: disparities in screening access in low socioeconomic status (SES) and African American individuals. A recent study by Anderson et al. shed light on decreased colon cancer screening in both African
American and low SES individuals discussing how 63.9\% of the cohort had never undergone colorectal cancer screening despite meeting criteria for screening recommendations based on age and family history of colorectal cancer [29]. Among these populations, diminished access to transportation or resources and inflexible work schedules can severely reduce patient turnout at appointments, as individuals report lower quality of healthcare and may be subject to implicit racial bias of their health care practitioners. It is possible such findings can be extrapolated for most gastrointestinal screenings in these high-risk groups. Attempts to increase screening access for these populations could prevent life threatening bowel complications.

Lastly, some studies have cited altered sensitivity to pain in schizophrenia may delay diagnosis and treatment of serious, life-threatening medical conditions [30-32]. Patients who are on antipsychotics may have an increased pain threshold, which makes it difficult for the patients to report somatic complaints later or less frequently [30-32]. Family caregivers also play an important role in taking care of these patients: in addition to managing unexpected behaviors of the patients such as aggression and violence, they often ensure completion of personal activities, chores (cooking, cleaning the house, etc.), other tasks (appointment arrangements, transportation, etc. ), and treatment compliance-all of which impose a heavy burden on them [33]. The results of several studies indicate that caregivers possess limited knowledge and awareness regarding the illness, the related coping strategies, and how to deal with and support the patient [33, 34]. The hours and commitment to caregiving activities that often lead to consequences for caregivers such as job loss, social activity decline imply that caregiver fatigue may contribute to late presentation of antipsychotic induced constipation. As in the case of our patient, the admission complaint was one that suggested an acute psychotic episode resulting from medication noncompliance, yet gathering collateral information from the caregiver revealed that there were issues in maintaining hygiene which the caregiver could not resolve on her own. 
Current recommendations on monitoring and prophylaxis of antipsychotic-induced constipation

While there is strong evidence that clozapine and olanzapine cause gut hypomotility, constipation, and even ileus, there is little consensus about how to best treat this. In an observational study done by Every-Palmer et al., there was reduction in the colonic transit time of clozapine-treated patients when treated with docusate and senna and macrogol according to the Porirua Protocol [9]. Chukhin et al. found that with orlistat use compared to placebo, the proportion of patients endorsing constipation reduced [35]. In addition to treatment with laxatives, a reduction of antipsychotic dose has been proposed in the literature, though this may not be feasible for a patient that has had multiple episodes of psychosis without the added risk of a relapse [36, 37]. Bulot et al. conducted a quasi-experimental trial by prescribing polyethylene glycol as a first-line laxative to inpatients receiving antipsychotic agents and found a three-fold reduction of severe constipation cases [36]. The prevention of this potentially life-threatening condition can be economic and well-tolerated by patients (Table 2).

There is some research into whether traditional herbal medications may be used as a supplement or even an alternative to laxative use to prevent constipation in those suffering from schizophrenia-associated constipation. Traditional Chinese medicines (TCMS) consist of acupuncture, tai chi, tuina massage, and botanical supplements. Historically, TCMs are under-utilized as a possible treatment option and even considered a form of pseudoscience due to concerns of herbal products having been contaminated with heavy metals, pesticides, and other toxic compounds, as well as the lack of clinical evidence with comparative randomized clinical trials with the standard care. Rathbone et al. showed that Chinese herbal medicines may be beneficial for people with schizophrenia when combined with antipsychotics. Though the sample size of the study was small, the study also showed that TCMs showed greater improvement in the treatment of IBS-C than cisapride and mosapride [39]. A study by Fan et al. in 2004 compared glycerol suppository with the traditional Chinese medicine (TCM) approaches of tuina massage and acupuncture for relieving constipation. 240 psychiatric inpatients were recruited, and for 14 days underwent either daily rectal enemas, daily periods of tuina massage, half an hour of acupuncture, or a combination of all three treatments. Compared to tuina massage, glycerol laxative was less effective in relieving constipation at both two days after treatment [40]. However, there were no data comparing the common pharmacological interventions for constipation, such as lactulose, polyethylene glycol, stool softeners, lubricant laxatives, or of novel treatments such as linaclotide.

Similarly, electroacupuncture in some studies is believed to be effective in treating opioid-induced constipation and certain functional GI diseases like dyspepsia, chronic constipation ( $\geq 1$ symptom including straining, hard stools, time-consuming defecation, sensations of incomplete evacuation, using manual assistance, for over 6 months). Yang et al. conducted a systematic review and meta-analysis of acupuncture treatment for opioidinduced constipation, which demonstrated that acupuncture has better results in clinical outcomes than non-acupuncture therapy [41]. Liu et al. conducted 28 sessions of electroacupuncture to increase the number of weekly complete spontaneous bowel movements (CSBMs) in 1075 patients with chronic severe functional constipation with no underlying pathologic cause for constipation. They found that eight weeks of EA increases CSBMs to 3 or more mean weekly CSBMs in the electro acupuncture group $37.7 \%$ compared with $14.1 \%$ in the placebo group [42]. Acupuncture-related adverse events during treatment were infrequent in both groups, and all were mild or transient and were considered safe for the treatment of chronic functional constipation, and thus has implications for use in patients who have schizophrenia and suffer from this condition.

\section{Conclusion}

Screening for constipation in schizophrenia may prove challenging due to poor communication abilities and disturbances in thought process. Current screening

Table 2 Comparison of efficacious laxative regimens [38]

\begin{tabular}{|c|c|c|}
\hline & MOA & Benefit \\
\hline Lactulose & Converts $\mathrm{NH} 3$ to $\mathrm{NH} 4+$ & Increases stool frequency by $40 \%$ \\
\hline $\begin{array}{l}\text { Polyethylene } \\
\text { glycol (MiraLAX) }\end{array}$ & $\begin{array}{l}\text { Osmotic agent, causes water retention in stool thereby } \\
\text { increasing stool frequency }\end{array}$ & $\begin{array}{l}\text { Most effective osmotic agent (especially without electrolytes), } \\
\text { palatability increases patient compliance, decreases incidence of fecal } \\
\text { impaction and increased stool frequency by } 60 \%\end{array}$ \\
\hline $\begin{array}{l}\text { Docusate } \\
\text { sodium }\end{array}$ & $\begin{array}{l}\text { Reduces surface tension of oil-water interface of stool } \\
\text { resulting in enhanced incorporation of water and fat }\end{array}$ & Overall baseline mean of 3.4 bowel movements \\
\hline Psyllium & $\begin{array}{l}\text { Soluble fiber that absorbs water in intestine that } \\
\text { promotes peristalsis and reduces transit time }\end{array}$ & $\begin{array}{l}\text { Better at increasing stool water content and total stool output in } \\
\text { comparison to docusate }\end{array}$ \\
\hline Senna & Induces peristaltic activity via enteric nerve stimulation & Fast acting \\
\hline
\end{tabular}


methods to assess constipation are subjective in nature and often labeled as a "patient report of constipation." Even if patients articulate his/her bowel habits, there are discrepancies surrounding the word constipation gastrointestinal, causing hypo-motility to go unreported and this runs the risk of evolving into life-threatening complications. Objective tests to gauge the severity and the presence of gastrointestinal hypo-motility often require the expertise of a GI specialist or are not cost-effective.

Prescribing antipsychotics should be accompanied by regular physical monitoring, appropriate use of laxatives, and early referral of constipated patients, before the development of pathologic gastrointestinal processes. Randomized clinical trials are needed to compare pharmacological and other interventions for antipsychotic-related constipation. Such trials should focus on the treatment of constipation, with an emphasis on patient tolerability and rapid resolution.

\section{Abbreviations}

FGA: First-generation antipsychotics; SGA: Second-generation antipsychotics

\section{Acknowledgements}

None.

\section{Authors' contributions}

AS developed the manuscript idea and edited the manuscript in its entirety. AV wrote the "Background" and "Conclusion" sections of the manuscript. SA wrote the "Case study" and "Abstract" sections of the manuscript. VC developed all the tables associated with the manuscript and obtained consent for publication. KF ensured the references were adequate and proofread the final version of manuscript. JN helped develop the initial idea behind the manuscript and revised the manuscript. The authors have read and approved the final manuscript.

\section{Funding}

None

\section{Availability of data and materials}

Not applicable.

\section{Declarations}

Ethics approval and consent to participate

Not applicable

\section{Consent for publication}

Not application

\section{Competing interests}

The authors declare that they have no competing interests.

Received: 21 February 2021 Accepted: 15 March 2021

Published online: 05 April 2021

\section{References}

1. De Hert M, Dockx L, Bernagie C, Peuskens B, Sweers K, Leucht S, Tack J, Van de Straete S, Wampers M, Peuskens J (2011) Prevalence and severity of antipsychotic related constipation in patients with schizophrenia: a retrospective descriptive study. BMC Gastroenterol 11(1):17. https://doi.org/1 $0.1186 / 1471-230 X-11-17$

2. Chen HK, Hsieh CJ (2018) Risk of gastrointestinal Hypomotility in schizophrenia and schizoaffective disorder treated with antipsychotics: a retrospective cohort study. Schizophr Res 195:237-244. https://doi.org/10.1 016/j.schres.2017.10.024
3. Koizumi T, Uchida H, Suzuki T, Sakurai H, Tsunoda K, Nishimoto M, Ishigaki T, Goto A, Mimura M (2013) Oversight of constipation in inpatients with schizophrenia: a cross-sectional study. Gen Hosp Psychiatry 35(6):649-652. https://doi.org/10.1016/i.genhosppsych.2013.06.007

4. Every-Palmer S, Newton-Howes G, Clarke MJ (2017) Pharmacological treatment for antipsychotic-related constipation. Cochrane Database Syst Rev 1(1):CD011128. https://doi.org/10.1002/14651858.CD011128.pub2

5. Stroup TS, Gray N (2018) Management of common adverse effects of antipsychotic medications. World Psychiatry 17(3):341-356. https://doi.org/1 0.1002/wps.20567

6. Saha, L., \& Shekhar Gautam, C. (2011). The effect of amlodipine alone and in combination with atenolol on bowel habit in patients with hypertension: an observation. Int Scholar Res Notices Gastroenterol, 2011, 757141. https://doi. org/10.5402/2011/757141, 3

7. Seeman P (2002) Atypical antipsychotics: mechanism of action. Can J Psychiatr 47(1):27-38 PMID: 11873706

8. Rehse M, Bartolovic M, Baum K, Richter D, Weisbrod M, Roesch-Ely D (2016) Influence of antipsychotic and anticholinergic loads on cognitive functions in patients with schizophrenia. Schizophr Res Treat 2016:8213165-8213110. https://doi.org/10.1155/2016/8213165

9. Every-Palmer S, Ellis PM, Nowitz M, Stanley J, Grant E, Huthwaite M et al (2017) The Porirua Protocol in the treatment of clozapine-induced gastrointestinal hypomotility and constipation: a pre- and post-treatment study. CNS Drugs 31(1):75-85. https://doi.org/10.1007/s40263-016-0391-y

10. Shirazi-Southall S, Rodriguez D, Nomikos G (2002) Effects of typical and atypical antipsychotics and receptor selective compounds on acetylcholine efflux in the hippocampus of the rat. Neuropsychopharmacol 26(5):583-594. https://doi.org/10.1016/S0893-133X(01)00400-6

11. Yogaratnam J, Biswas N, Vadivel R, Jacob R (2013) Metabolic complications of schizophrenia and antipsychotic medications--an updated review. East Asian Arch Psychiatr 23(1):21-28

12. Dauwan M, Begemann MJ, Heringa SM, Sommer IE (2016) Exercise improves clinical symptoms, quality of life, global functioning, and depression in schizophrenia: a systematic review and meta-analysis. Schizophr Bull 42(3): 588-599. https://doi.org/10.1093/schbul/sbv164

13. Saedisomeolia A, Djalali M, Moghadam AM, Ramezankhani O, Najmi L (2011) Folate and vitamin B12 status in schizophrenic patients. J Res Med Sci 16(Suppl 1):S437-S441 PMID: 22247731; PMCID: PMC3252772

14. Jakobsen AS, Speyer H, Nørgaard HC, Karlsen M, Hjorthøj C, Krogh J et al (2018) Dietary patterns and physical activity in people with schizophrenia and increased waist circumference. Schizophr Res 199:109-115. https://doi. org/10.1016/.jschres.2018.03.016

15. Küçükerdönmez Ö, Urhan M, Alıın M, Hacıraifoğlu Ö, Yıldız B (2019) Assessment of the relationship between food addiction and nutritional status in schizophrenic patients. Nutr Neurosci 22(6):392-400. https://doi. org/10.1080/1028415X.2017.1392429 Epub 2017 Oct 27. PMID: 29078744

16. Kim EJ, Lim SY, Lee HJ, Lee JY, Choi S, Kim SY, Kim JM, Shin IS, Yoon JS, Yang SJ, Kim SW (2017) Low dietary intake of n-3 fatty acids, niacin, folate, and vitamin $\mathrm{C}$ in Korean patients with schizophrenia and the development of dietary guidelines for schizophrenia. Nutr Res 45:10-18. https://doi.org/1 0.1016/j.nutres.2017.07.001 Epub 2017 Jul 19. PMID: 29037327

17. Dickerson F, Gennusa JV, Stallings C, Origoni A, Katsafanas E, Sweeney K et al (2020) Protein intake is associated with cognitive functioning in individuals with psychiatric disorders. Psychiatry Res 284:112700. https://doi. org/10.1016/.jpsychres.2019.112700

18. Constipation screening in people taking clozapine: a diagnostic accuracy study. (2020, June 1). ScienceDirect. https://www.sciencedirect.com/ science/article/pii/S0920996420301389

19. Osterman MT, Foley C, Matthias I (2017) Clozapine-induced acute gastrointestinal necrosis: a case report. J Med Case Rep 11(1):270. https:// doi.org/10.1186/s13256-017-1447-4

20. Hibbard KR, Propst A, Frank DE, Wyse J (2009) Fatalities associated with clozapine-related constipation and bowel obstruction: a literature review and two case reports. Psychosomatics 50(4):416-419. https://doi.org/10.11 76/appi.psy.50.4.416

21. Palmer SE, McLean RM, Ellis PM, Harrison-Woolrych M (2008) Lifethreatening clozapine-induced gastrointestinal hypomotility: an analysis of 102 cases. J Clin Psychiatry 69(5):759-768. https://doi.org/10.4088/jcp. v69n0509

22. Shirazi A, Stubbs B, Gomez L, Moore S, Gaughran F, Flanagan RJ, MacCabe JH, Lally J (2016) Prevalence and predictors of clozapine-associated 
constipation: a systematic review and meta-analysis. Int J Mol Sci 17(6):863. https://doi.org/10.3390/ijms17060863

23. Stanniland C, Taylor D (2000) Tolerability of atypical antipsychotics. Drug Saf 22(3):195-214. https://doi.org/10.2165/00002018-200022030-00004

24. Virtanen T, Eskelinen S, Sailas E, Suvisaari J (2017) Dyspepsia and constipation in patients with schizophrenia spectrum disorders. Nordic $J$ Psychiatry 71(1):48-54. https://doi.org/10.1080/08039488.2016.1217044

25. Werner S, Malaspina D, Rabinowitz I (2007) Socioeconomic status at birth is associated with risk of schizophrenia: population based multilevel study. Schizophr Bull 33(6):1373-1378. https://doi.org/10.1093/schbul/sbm032

26. Holmberg SK, Kane C (1999) Health and self-care practices of persons with schizophrenia. Psychiatr Serv 50(6):827-829. https://doi.org/10.1176/ps.50.6.827

27. Taş, S. (2017). Early period self-care ability and care requirements of schizophrenia patients after discharge. Journal of Psychiatric Nursing. doi:10. 14744/phd.2017.64935

28. Çiftçi B, Yıldırım N, Şahin Altun Ö, Avşar G (2015) What level of self care agency in mental illness? The factors affecting self-care agency and self-care agency in patients with mental illness. Arch Psychiatr Nurs 29(6):372-376. https://doi.org/10.1016/j.apnu.2015.06.007

29. Andersen SW et al (2019) Association of race and socioeconomic status with colorectal cancer screening, colorectal cancer risk, and mortality in Southern US adults. JAMA Netw Open 2:12. https:/doi.org/10.1001/jamanetworkopen.2019.17995

30. Guieu R, Samuélian JC, Coulouvrat H (1994) Objective evaluation of pain perception in patients with schizophrenia. Brit J Psychiatry 164(2):253-255. https://doi.org/10.1192/bjp.164.2.253

31. Engels G, Francke AL, van Meijel B, Douma JG, de Kam H, Wesselink W, Houtjes W, Scherder EJA (2014) Clinical pain in schizophrenia: a systematic review. J Pain 15(5):457-467. https://doi.org/10.1016/j.jpain.2013.11.005

32. Thrush A, Hyder AA (2014) The neglected burden of caregiving in low- and middle-income countries. Disability Health J 7(3):262-272. https://doi.org/1 0.1016/j.dhjo.2014.01.003

33. Durmaz H, Okanlı A (2014) Investigation of the effect of self-efficacy levels of caregiver family members of the individuals with schizophrenia on burden of care. Arch Psychiatr Nurs 28(4):290-294. https://doi.org/10.1016/j.apnu.2014.04.004

34. Gater A, Rofail D, Tolley C, Marshall C, Abetz-Webb L, Zarit SH et al (2014) "Sometimes it's difficult to have a normal life": results from a qualitative study exploring caregiver burden in schizophrenia. Schizophr Res Treat 2014:368215

35. Chukhin E, Takala P, Hakko H, Raidma M, Putkonen H, Rasanen P et al (2013) In a randomized placebo-controlled add-on study orlistat significantly reduced clozapine-induced constipation. Int Clin Psychopharmacol 28(2):6770. https://doi.org/10.1097/YIC.0b013e32835b08d2

36. Bulot V, Lemogne C, Nebot N, Blondon H, Roux P (2016) Systematic prevention of severe constipation induced by antipsychotic agents: a quasiexperimental study. Eur Neuropsychopharmacol 26(10):1690-1691. https:// doi.org/10.1016/i.euroneuro.2016.08.004

37. Every-Palmer S et al (2020) Constipation screening in people taking clozapine: a diagnostic accuracy study. Schizophr Res 220:179-186. https:// doi.org/10.1016/j.schres.2020.03.032

38. Paré P, Fedorak RN (2014) Systematic review of stimulant and nonstimulant laxatives for the treatment of functional constipation. Canadian J Gastroenterol Hepatol 28(10):549-557. https://doi.org/10.1155/2014/631740

39. Rathbone J, Zhang L, Zhang M, Xia J, Liu X, Yang Y (2005) Chinese herbal medicine for schizophrenia. Cochrane Database Syst Rev 2005(4):CD003444. https://doi.org/1 0.1002/14651858.CD003444.pub2 PMID: 16235320; PMCID: PMC7017283

40. Fan Y, Luo W (2004) Clinical study on treatment of constipation caused by antipsychotic drugs with acupuncture and Tuina combined with laxative suppository. J Acupuncture Tuina Sci 2(3):51-54

41. Liu Z, Yan S, Wu J, He L, Li N, Dong G, Fang J, Fu W, Fu L, Sun J, Wang L, Wang S, Yang J, Zhang H, Zhang J, Zhao J, Zhou W, Zhou Z, Ai Y, Zhou K, Liu J, Xu H, Cai Y, Liu B (2016) Acupuncture for chronic severe functional constipation: a randomized trial. Ann Intern Med 165(11):761-769. https:// doi.org/10.7326/M15-3118

42. Yang P, Wang Y, Xiao Y, Ma Q, Ma R, Mi J, Hui J (2020) Acupuncture for opioid-induced constipation: protocol for a systematic review and metaanalysis. Medicine (Baltimore) 99(49):e23352. https://doi.org/10.1097/MD. 0000000000023352

\section{Publisher's Note}

Springer Nature remains neutral with regard to jurisdictional claims in published maps and institutional affiliations.

\section{Submit your manuscript to a SpringerOpen ${ }^{\circ}$ journal and benefit from:}

- Convenient online submission

- Rigorous peer review

- Open access: articles freely available online

- High visibility within the field

- Retaining the copyright to your article

Submit your next manuscript at $\boldsymbol{\nabla}$ springeropen.com 